\title{
Tailored biosensors for drug screening, efficacy assessment and toxicity evaluation
}

$$
\text { Yi Tao*a, Lin Chena, Meiling Panª, Fei Zhua, Dong Zhu*b }
$$

${ }^{a}$ College of Pharmaceutical Science, Zhejiang University of Technology, Hangzhou, 310014, China

${ }^{b}$ School of Pharmacy, Nanjing University of Chinese Medicine, Nanjing, Jiangsu, 210023, PR China 
Table S1. The strengths and limitations of current biosensors for drug discovery

\begin{tabular}{|c|c|c|c|c|c|c|}
\hline Name & $\begin{array}{l}\text { Transduction } \\
\text { mechanism }\end{array}$ & $\begin{array}{l}\text { Typical material } \\
\text { requirements }\end{array}$ & Strengths & Limitations & Applications & Ref. \\
\hline \multirow[t]{2}{*}{ SPR biosensor } & $\begin{array}{l}\text { Microfluidic } \\
\text { surface-based } \\
\text { method }\end{array}$ & $\begin{array}{l}\text { (1) } \sim 15 \mathrm{nmol} \text { protein for assay } \\
\text { development and screening of } \\
2,000 \text { compounds } \\
\text { (2) Reference compounds for } \\
\text { evaluation of protein } \\
\text { functionality }\end{array}$ & $\begin{array}{l}\text { (1) Direct time-resolved } \\
\text { determination of interactions } \\
\text { over a broad range of } \\
\text { conditions } \\
\text { (2) High sensitivity allows } \\
\text { analysis of fragments }\end{array}$ & $\begin{array}{l}\text { (1) Requires immobilization of functional } \\
\text { target with high stability over time and } \\
\text { towards regeneration procedures } \\
\text { (2) Signals affected by solvent effects }\end{array}$ & Drug screening & (2) \\
\hline & $\begin{array}{l}\text { Microarray-based } \\
\text { method with CCD } \\
\text { camera detection }\end{array}$ & $\begin{array}{l}\text { (1) } \sim 100-150 \mathrm{nmol} \text { protein } \\
\text { (2) Reference compounds to } \\
\text { assess binding specificity or to } \\
\text { identify allosteric binders }\end{array}$ & $\begin{array}{l}\text { (1) Very high sensitivity } \\
\text { (2) Ultra-high throughput }\end{array}$ & $\begin{array}{l}\text { (1) Requires chemical modification of } \\
\text { compounds to immobilize ligands through } \\
\text { a covalent linker to a surface preferably in } \\
\text { different orientations } \\
\text { (2) Detergents are not tolerated }\end{array}$ & Drug screening & (2) \\
\hline $\begin{array}{l}\text { Optical waveguide grating } \\
\text { biosensor }\end{array}$ & $\begin{array}{l}\text { Microplate based } \\
\text { method }\end{array}$ & $\begin{array}{l}\text { (1) Target proteins or cells } \\
\text { immobilized on the plate surface }\end{array}$ & $\begin{array}{l}\text { (1) High-throughput } \\
\text { (2) Ability to measure weak } \\
\text { binders }\end{array}$ & $\begin{array}{l}\text { (1) Only allow for Kd determination } \\
\text { (2) Low sensitivity } \\
\text { (3) High protein consumption }\end{array}$ & Drug screening & $(22)$ \\
\hline $\begin{array}{l}\text { Biolayer interferometry } \\
\text { biosensor }\end{array}$ & Tip based method & $\begin{array}{l}\text { (1) Target proteins immobilized } \\
\text { on the tip surface } \\
\text { (2) Requires a minimum sample } \\
\text { volume of } 80 \mu \mathrm{L} \text { in plate }\end{array}$ & $\begin{array}{l}\text { (1) Minimally perturbed by } \\
\text { changes in the medium } \\
\text { (2) A range of biosensor-tips } \\
\text { enable analyte evaluations in } \\
\text { parallel }\end{array}$ & $\begin{array}{l}\text { (1) Low sensitivity } \\
\text { (2) High reagent consumption }\end{array}$ & Drug screening & $(9,27)$ \\
\hline $\begin{array}{l}\text { Grating-coupled } \\
\text { interferometry } \\
\text { biosensor }\end{array}$ & $\begin{array}{l}\text { Sensor chip-based } \\
\text { method }\end{array}$ & $\begin{array}{l}\text { (1) Target proteins immobilized } \\
\text { on the surface of the chip } \\
\text { (2) A reference light beam is }\end{array}$ & $\begin{array}{l}\text { (1) High sensitivity (reliable } \\
\text { kinetics below } 0.01 \mathrm{pg} \cdot \mathrm{mm}^{-2} \text { ) } \\
\text { (2) No-clog microfluidics }\end{array}$ & $\begin{array}{l}\text { (1) The ligand may bind to the chip } \\
\text { surface }\end{array}$ & Drug screening & (33) \\
\hline
\end{tabular}


needed to measure the phase

change reliably and precisely

$\begin{array}{ll}\text { Switchable DNA nanolevers } & \text { Microfluidic } \\ \text { biosensor } & \text { biochip-based } \\ & \text { method }\end{array}$

(1) The ligand molecule is

(1) High content analysis crosslinked to the DNA strand

(2) The coupled ligand should

be purified from any free DNA

(3) One measurement requires

$40 \mu \mathrm{L}$ of $250 \mathrm{nM}$ ligand-DNA

conjugate

\section{Fluorescent biosensor}

Förster resonance

energy transfer

(FRET)

\section{$(1) \geqslant 30 \%$ overlap between}

emission spectrum of the donor

and the excitation spectrum of

the acceptor

(2) Close distance (1-10 nm)

between the donor and the

acceptor

Aggregation-induced (1) Require specific AIEgens emission (AIE)

Near infrared

(1) Require specific NIR

fluorescence (NIR) fluorophore

Electrochemical

signal transduction

can occur through

(working, reference and counter

electrodes)

(2) An electrochemical
(2) Requires low sample

volumes $(50 \mu \mathrm{L})$

(1) The ability to perform biochemical assays in real time in living cells

(2) Inexpensive and can be performed on standard wide field microscopes

(1)No aggregation-caused quenching effect

(1) Reduced tissue autofluorescence

(2) Deep tissue penetration

(1) High sensitivity

(2) Low cost

(3) Simple instrumentation (4) Portability
(1) The protein may bind non-specifically Drug screening to double stranded DNA

(2) Not ideal for high-throughput

screening due to the relatively slow chip regeneration

(1) FRET efficiency can be quite low, and Drug screening Efficacy assessment false positives and false negatives must be controlled Toxicity evaluation

(2) Biosensors using GFPs as donors and acceptors require careful characterization, because the bulky GFP-tag can affect protein function

(1) Require several synthesis steps to

Drug screening synthesize and modify AIEgens

Efficacy assessmen Toxicity evaluation

(1) Light scattering and tissue absorption Drug screening (2) Need to control optical properties, Efficacy assessment Toxicity evaluation (1) Fabrication of the electrode consumes Drug screening 
current, potential,

workstation

conductance, or field

effect

Photoelectrochemical

A suitable

photoactive

(1) An excitation source

(2) A monochromator

(3) An electrochemical

nanomaterial

workstation

(4) Three electrode system

and charge

separation occurs as

(working, reference and counter

the electron-hole electrodes)
(1) High sensitivity

(2) Simple

(3) Cheap

(4) Easy to miniaturize

Drug screening

subject to low photo-to-current conversion

efficiencies

(2) Susceptibilities to photobleaching

(3) Electrode fouling

pair formed by the

excitation of the

electron from the

valence to the

conductive band 
Table S2. Key examples of SPR biosensors for drug discovery

\begin{tabular}{|c|c|c|c|c|c|c|}
\hline Drug target & $\begin{array}{c}\text { Immobilization } \\
\text { method }\end{array}$ & $\begin{array}{c}\text { Detection time } \\
\text { per sample }\end{array}$ & Optical platform & Drugs & $\begin{array}{c}\text { LOD/linear } \\
\text { range }\end{array}$ & Ref. \\
\hline Metallo- $\beta$-lactamase & $\begin{array}{l}\text { streptavidin-biot } \\
\text { in }\end{array}$ & $7 \mathrm{~min}$ & Prism-based using gold film & 490 fragments & $\begin{array}{c}0.2 \mu \mathrm{M} / 0.2-100 \\
\mu \mathrm{M}\end{array}$ & $(10)$ \\
\hline Clostridial collagenases & EDC/NHS & $3 \mathrm{~min}$ & Prism-based using gold film & $\begin{array}{l}\text { Mercaptoacetamide } \\
\text { Compounds }\end{array}$ & $100 \mu \mathrm{M}$ & (11) \\
\hline TNF & EDC/NHS & $4 \mathrm{~min}$ & Prism-based using gold film & $\begin{array}{c}\text { Physcion- } 8-O-\beta-D-m o n o g l u c \\
\text { oside }\end{array}$ & $\begin{array}{c}40 \mathrm{nM} / 40 \mathrm{nM}- \\
4 \mu \mathrm{M}\end{array}$ & $(12)$ \\
\hline STAT3 & EDC/NHS & $4 \mathrm{~min}$ & Prism-based using gold film & Static & $\begin{array}{c}0.5 \mu \mathrm{M} / 0.5-100 \\
\mu \mathrm{M}\end{array}$ & (13) \\
\hline CXCR4 & EDC/NHS & $4 \mathrm{~min}$ & Prism-based using gold film & AMD 3100, geftinib, & $7.8 \mathrm{nM} / 7.8$ & (14) \\
\hline
\end{tabular}




\begin{tabular}{|c|c|c|c|c|c|c|}
\hline & & & & senkyunolide I & nM-64 $\mu \mathrm{M}$ & \\
\hline$\alpha$-Glucosidase & co-precipitation & $40 \mathrm{~min}$ & Silver nanoplates & Acarbose & $\begin{array}{c}5 \mathrm{nM} / 0.05 \\
\mu \mathrm{M}-100 \mu \mathrm{M}\end{array}$ & (15) \\
\hline FKBP12 & EDC/NHS & $10 \mathrm{~min}$ & $\begin{array}{l}\text { 3D-dextran hydrogel coated } \\
\text { gold film }\end{array}$ & FK506, SB203580 & $10 \mathrm{mM}$ & (16) \\
\hline PPP1R15B & $\begin{array}{l}\text { streptavidin-biot } \\
\text { in }\end{array}$ & $3 \min$ & Prism-based using gold film & GBZ, sephin 1 , raphin $1, \mathrm{C} 3$ & $\begin{array}{c}0.02 \\
\mu \mathrm{M} / 0.02-25\end{array}$ & (17) \\
\hline $\mathrm{PI} 3 \mathrm{~K} \gamma$ & EDC/NHS & $6 \mathrm{~min}$ & Prism-based using gold film & $\mathrm{AZ1-AZ4}$ & $\begin{array}{c}\mu \mathrm{M} \\
4 \mathrm{nM} / 4 \\
\mathrm{nM}-1000 \mathrm{nM}\end{array}$ & (18) \\
\hline Xanthine oxidase & EDC/NHS & $17 \mathrm{~min}$ & Prism-based using gold film & Allopurinol & $\begin{array}{c}3.1 \mu \mathrm{M} / 3.1 \mu \mathrm{M}- \\
200 \mu \mathrm{M}\end{array}$ & (19) \\
\hline DNA Methyltransferase & EDC/NHS & $6 \mathrm{~h}$ & ds-DNA-covered gold film & 5-Aza (ribose nucleoside) & $\begin{array}{c}0.1 \mu \mathrm{M} / 0.1 \mu \mathrm{M} \\
-15 \mu \mathrm{M}\end{array}$ & (20) \\
\hline
\end{tabular}

Table S3. Selected cases of optical waveguide grating biosensors for drug discovery

\begin{tabular}{|c|c|c|c|c|}
\hline Drug target & Optical platform & Drugs & LOD/linear range & Ref. \\
\hline Muscarinic M3 Receptor & Corning Epic & $\begin{array}{l}\text { Atropine, ipratropium, } \\
\text { N-methyl-scopolamine, } \\
\text { tiotropium }\end{array}$ & $10^{-11} \mathrm{M} / 10^{-11}-10^{-6} \mathrm{M}$ & (23) \\
\hline $\begin{array}{l}\text { Muscarinic acetylcholine M2 } \\
\text { receptor }\end{array}$ & Corning Epic & $\begin{array}{l}\text { Scopolamine, hyoscyamine, } \\
\text { chelerythrine, sanguinarine }\end{array}$ & & (24) \\
\hline
\end{tabular}




$\begin{array}{cccc}\begin{array}{c}\text { G protein-activated inwardly } \\ \text { rectifying potassium channels } \\ \text { Cannabinoid receptors }\end{array} & \text { Corning Epic } & \text { ML297 analogs } & \text { (25) } \\ & \text { Corning Epic } & \begin{array}{c}\text { CP55940, WIN55, } \\ \text { SR141716A }\end{array} & \text { 100 nM/100 nM-100 } \mu \mathrm{M}\end{array}$


Table S4. Biolayer interferometry biosensors for drug discovery

\begin{tabular}{|c|c|c|c|c|c|}
\hline Drug target & $\begin{array}{l}\text { Detection time } \\
\text { per sample }\end{array}$ & $\begin{array}{l}\text { Optical } \\
\text { platform }\end{array}$ & Drugs & LOD/linear range & Ref. \\
\hline $\begin{array}{c}\text { Glutamate } \\
\text { carboxypeptidase II }\end{array}$ & $40 \mathrm{~min}$ & $\begin{array}{l}\text { ForteBio Octet } \\
\text { Red }\end{array}$ & СТT54, СТT54.2 & $100 \mu \mathrm{M}$ & $(28)$ \\
\hline Glutaminase & $10 \mathrm{~min}$ & ForteBio K2 & Ebselen & $0.1 \mu \mathrm{M} / 0.1 \mu \mathrm{M}-10 \mu \mathrm{M}$ & (29) \\
\hline Double-stranded DNA & $2 \min$ & $\begin{array}{l}\text { ForteBio Octet } \\
\text { Red }\end{array}$ & $\begin{array}{l}\text { Actinomycin D, Doxorubicin, } \\
\text { echinomycin, actinomycin V, chartreusin }\end{array}$ & $\begin{array}{c}0.03 \mu \mathrm{M} / 0.03 \mu \mathrm{M}-5 \\
\mu \mathrm{M}\end{array}$ & (30) \\
\hline $\begin{array}{l}\text { Tissue } \\
\text { transglutaminase and } \\
\text { fibronectin interaction }\end{array}$ & $4.5 \mathrm{~min}$ & Pall ForteBio & TG53 analogues & $16 \mathrm{nM} / 16 \mathrm{nM}-440 \mu \mathrm{M}$ & (31) \\
\hline $\begin{array}{l}\text { SARSCoV-2 RBD and } \\
\text { hACE2 interaction }\end{array}$ & $10 \mathrm{~min}$ & FortebBio & Corilagin & $\begin{array}{c}3.13 \mu \mathrm{M} / 3.13 \\
\mu \mathrm{M}-100 \mu \mathrm{M}\end{array}$ & (32) \\
\hline
\end{tabular}


Table S5. Recent applications of grating coupled interferometry biosensors for drug discovery

\begin{tabular}{cccccc}
\hline Drug target & $\begin{array}{c}\text { Detection time } \\
\text { per sample }\end{array}$ & Optical platform & Drugs & $\begin{array}{c}\text { LOD/linear } \\
\text { range }\end{array}$ & Ref. \\
\hline Bovine serum albumin & $160 \mathrm{~s}$ & Creoptix WAVE & Epigallocatechin- & $0.533 \mu \mathrm{M} /$ & $(33)$ \\
\hline
\end{tabular}


gallate (EGCG) $\quad 0.533 \mu \mathrm{M}-17.5$

$\mathrm{mM}$

Carbonic anhydrase II

Small molecules

$10 \mu \mathrm{M}-50 \mu \mathrm{M}$ library 
Table S6. Several important FRET biosensors for drug discovery

\begin{tabular}{|c|c|c|c|c|c|c|c|}
\hline Drug Target & Fluorophore (D/A) & $\lambda_{\mathrm{ex}}(\mathbf{n m})$ & $\lambda_{\text {em }}(\mathbf{n m})$ & Drugs & LOD & Linear range & Ref. \\
\hline $\mathrm{AChE}$ & AuNCs@11-MUA/Cu² (quencher) & 286 & 608 & Tacrine & $1 \mathrm{nM}$ & $1-100 \mathrm{nM}$ & $(40)$ \\
\hline TYR & Graphitic $\mathrm{C}_{3} \mathrm{~N}_{4} /$ melanin-like polymer & 310 & 438 & Kojic acid & $0.78 \mu \mathrm{g} / \mathrm{mL}$ & $0.78 \mu \mathrm{g} / \mathrm{mL}-1000 \mu \mathrm{g} / \mathrm{mL}$ & $(41)$ \\
\hline TYR & $\begin{array}{l}\text { Covalent organic nanospheres/ } \\
\text { melanin-like polymer (quencher) }\end{array}$ & 432 & 580 & Kojic acid & $0.001 \mu \mathrm{g} / \mathrm{mL}$ & $0.001 \mu \mathrm{g} / \mathrm{mL}-1200 \mu \mathrm{g} / \mathrm{mL}$ & $(42)$ \\
\hline HIV-1 RT & Tetramethylrhodamine/Cy5 & 540 & 580 & Zidovudine, Nevirapine & $\begin{array}{l}0.625 \mu \mathrm{M} / \\
20 \mathrm{nM}\end{array}$ & $\begin{array}{c}0.625 \mu \mathrm{M}-20 \mu \mathrm{M} / \\
20 \mathrm{nM}-500 \mathrm{nM}\end{array}$ & $(43)$ \\
\hline$\alpha$-Glucosidase & N-doped CDs/CoOOH nanoflakes & 405 & 510 & Acarbose & $10^{-9} \mathrm{M}$ & $10^{-9} \mathrm{M}-10^{-3} \mathrm{M}$ & $(44)$ \\
\hline Cereblon & Trp/MANT & 295 & 440 & Thalidomide & $0.1 \mu \mathrm{M}$ & $0.1-40 \mu \mathrm{M}$ & $(45)$ \\
\hline
\end{tabular}




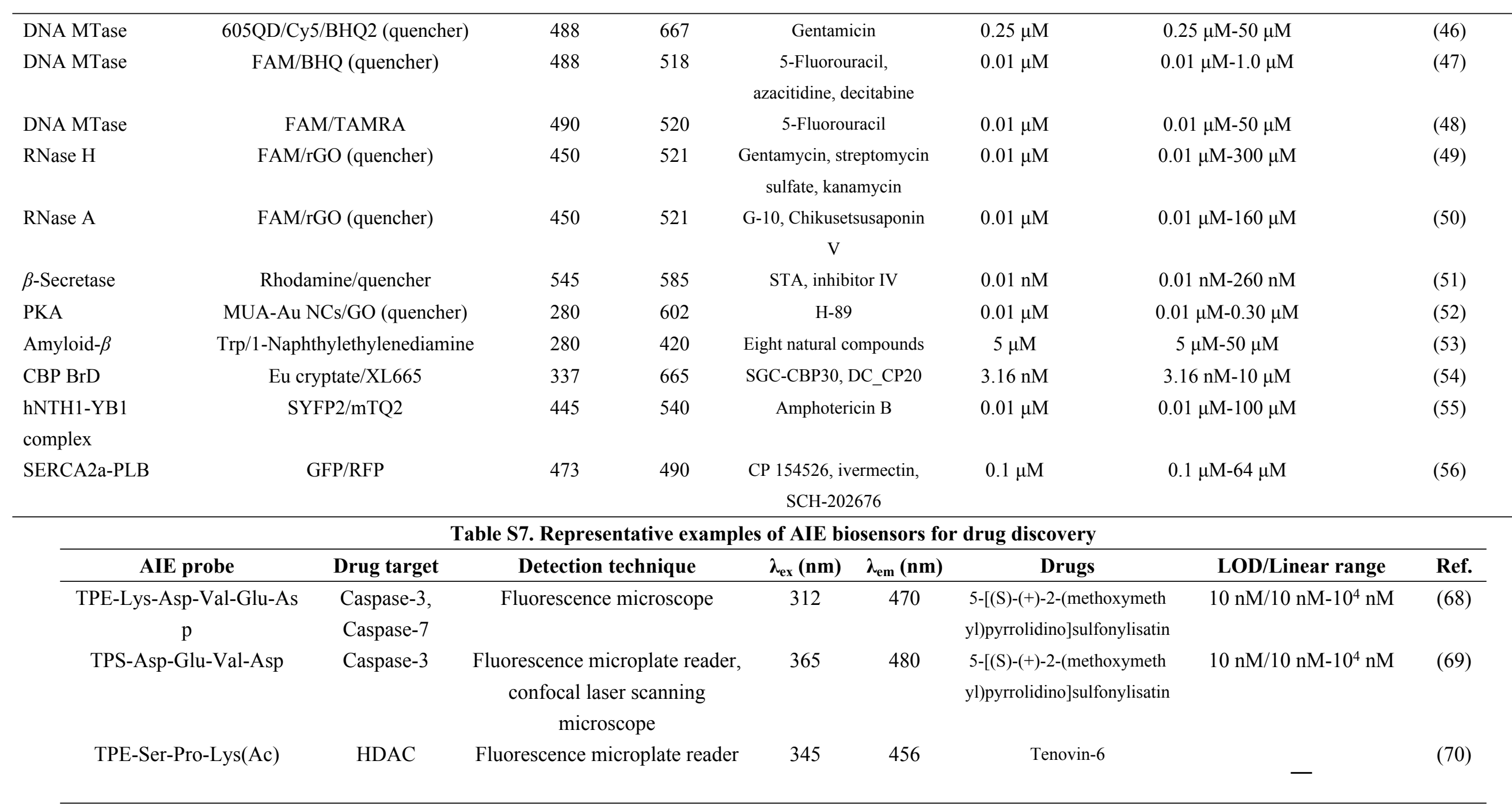




\begin{tabular}{|c|c|c|c|c|c|c|c|c|c|}
\hline TPE-Ser-Asp-Lys-Pro & $\mathrm{ACE}$ & $\begin{array}{r}\text { Microplate } \\
\mathrm{n}\end{array}$ & $\begin{array}{l}\text { ader, Fluorescence } \\
\text { croscope }\end{array}$ & 320 & 470 & Captopril & \multicolumn{2}{|c|}{$0.5 \mathrm{nM} / 0.5-50 \mathrm{nM}$} & $(71)$ \\
\hline TPE-Py & Telomerase & \multicolumn{2}{|c|}{$\begin{array}{l}\text { Laser scanning confocal } \\
\text { microscopy }\end{array}$} & 405 & 575 & $\begin{array}{c}\text { 3'-Azido-3'-deoxythymidine } \\
\text {, epigallocatechin gallate }\end{array}$ & \multicolumn{2}{|c|}{$0.01 \mu \mathrm{M} / 0.01-20 \mu \mathrm{M}$} & $(72)$ \\
\hline $\begin{array}{c}\text { TPE-Gly-Lys(Ac)-Tyr- } \\
\text { Asp-Asp }\end{array}$ & SIRT1 & \multicolumn{2}{|c|}{$\begin{array}{l}\text { Fluorescence microplate reader, } \\
\text { Laser scanning confocal } \\
\text { microscope }\end{array}$} & 320 & 465 & EX527, SRT1720 & \multicolumn{2}{|c|}{$10 \mathrm{nM} / 10 \mathrm{nM}-2000 \mathrm{nM}$} & (73) \\
\hline t-TPEM & $\begin{array}{l}\text { Monoamine } \\
\text { oxidase-A }\end{array}$ & \multicolumn{2}{|c|}{$\begin{array}{l}\text { Laser scanning confocal } \\
\text { microscopy }\end{array}$} & 360 & 560 & Clorgyline & & & $(74)$ \\
\hline TPE-Lys-Phe-Pro-Glu & DPP-4 & \multicolumn{2}{|c|}{$\begin{array}{l}\text { Fluorescence microplate reader, } \\
\text { Laser scanning confocal } \\
\text { microscope }\end{array}$} & 320 & 450 & Diprotin A & \multicolumn{2}{|c|}{$\begin{array}{c}0.005 \mu \mathrm{M} / 0.005 \mu \mathrm{M}-100 \\
\mu \mathrm{M}\end{array}$} & $(75)$ \\
\hline TPETH-DDYVADC & Caspase-1 & \multicolumn{2}{|c|}{ Fluorescence microplate reader } & 420 & 630 & Coumarin & & & $(76)$ \\
\hline TPE4S & Sialidases & \multicolumn{2}{|c|}{ Microplate reader } & 380 & 630 & $\begin{array}{l}\text { Zanamivir, oseltamivir } \\
\text { acid, oseltamivir }\end{array}$ & \multicolumn{2}{|c|}{$7.5 \mu \mathrm{M} / 7.5 \mu \mathrm{M}-1000 \mu \mathrm{M}$} & $(77)$ \\
\hline \multicolumn{10}{|c|}{ Table S8. Other fluorescent biosensors for drug discovery } \\
\hline Drug Target & Fluorophore & $\lambda_{\mathrm{ex}}(\mathrm{nm})$ & $\lambda_{\mathrm{em}}(\mathbf{n m})$ & \multicolumn{2}{|c|}{ Drugs } & \multicolumn{2}{|c|}{ LOD/Linear range } & Ref. & \\
\hline ER & Acrylodan & 386 & 451 & \multicolumn{2}{|c|}{$\begin{array}{l}\text { 4-Hydroxytamoxifen, } \\
\text { diethylstilbestrol, E2 }\end{array}$} & \multicolumn{3}{|c|}{$10^{-4} \mu \mathrm{M} / 10^{-4} \mu \mathrm{M}-20 \mu \mathrm{M}$} & \\
\hline GGT & HXPI & 680 & 708 & \multicolumn{2}{|c|}{ Acivicin, DON } & \multicolumn{2}{|c|}{$10^{-3} \mathrm{mM} / 10^{-3} \mathrm{mM}-10 \mathrm{mM}$} & $(80)$ & \\
\hline$\beta$-Glucuronidase & DDAO-glu & 605 & 660 & \multicolumn{2}{|c|}{ Baicalin } & \multicolumn{2}{|l|}{-} & $(81)$ & \\
\hline MMP & Cy3 & 488 & 590 & \multicolumn{2}{|c|}{ SB-3CT } & \multicolumn{2}{|c|}{$0.1 \mathrm{nM} / 0.1 \mathrm{nM}-1000 \mathrm{nM}$} & $(82)$ & \\
\hline HAT & Thioflavin T-G4 & 425 & 485 & \multicolumn{2}{|c|}{ C464, EX 527} & \multicolumn{2}{|c|}{$0.1 \mu \mathrm{M} / 0.1 \mu \mathrm{M}-60 \mu \mathrm{M}$} & $(83)$ & \\
\hline
\end{tabular}




\begin{tabular}{|c|c|c|c|c|c|c|}
\hline ALDH & TBAs & 450 & 642 & Disulfiram & - & $(84)$ \\
\hline Hsp 90 & CdTe QD & 488 & 640 & Celastrol & - & (85) \\
\hline FAP $\alpha$ & $\mathrm{CyOH}$ & 680 & 710 & $\begin{array}{l}\text { Pirfenidone, rapamycin, decorin, } \\
\text { simvastatin }\end{array}$ & - & (86) \\
\hline TYR & HB-NP & 480 & 675 & Kojic acid & $0.01 \mu \mathrm{M} / 0.01 \mu \mathrm{M}-200 \mu \mathrm{M}$ & (87) \\
\hline TYR & PMNPs & 365 & 525 & Kojic acid & $1 \mu \mathrm{M} / 1 \mu \mathrm{M}-100 \mu \mathrm{M}$ & (88) \\
\hline GLS1 & FITC & 486 & 529 & BPTES, CB839 & $0.002 \mu \mathrm{M} / 0.002 \mu \mathrm{M}-50 \mu \mathrm{M}$ & (89) \\
\hline ENPP1 & $\begin{array}{l}\text { Tokyo Green } \\
\text { (TG)-mAMP }\end{array}$ & 485 & 535 & AMP & $10^{-8} \mathrm{M} / 10^{-8} \mathrm{M}-10^{-3} \mathrm{M}$ & $(90)$ \\
\hline $\begin{array}{c}\text { Sirtuin } \\
\text { defatty-acylase }\end{array}$ & Dabcyl-PH & 485 & 535 & Nicotinamide & $10^{-6} \mathrm{M} / 10^{-6} \mathrm{M}-10^{-3} \mathrm{M}$ & (91) \\
\hline HDAC & FITC & 488 & 520 & Trichostatin A & $10^{-3} \mathrm{nM} / 10^{-3} \mathrm{nM}-10^{4} \mathrm{nM} /$ & (91) \\
\hline PTP1B & Cy5 & 650 & 670 & Sodium orthovanadate & $0.01 \mu \mathrm{M} / 0.01 \mu \mathrm{M}-10 \mu \mathrm{M}$ & $(92)$ \\
\hline PGA & AMNA & 405 & 540 & Oleanolic acid & $10 \mu \mathrm{M} / 10 \mu \mathrm{M}-100 \mu \mathrm{M}$ & (93) \\
\hline
\end{tabular}

Table S9. A list of electrochemical biosensors for drug discovery

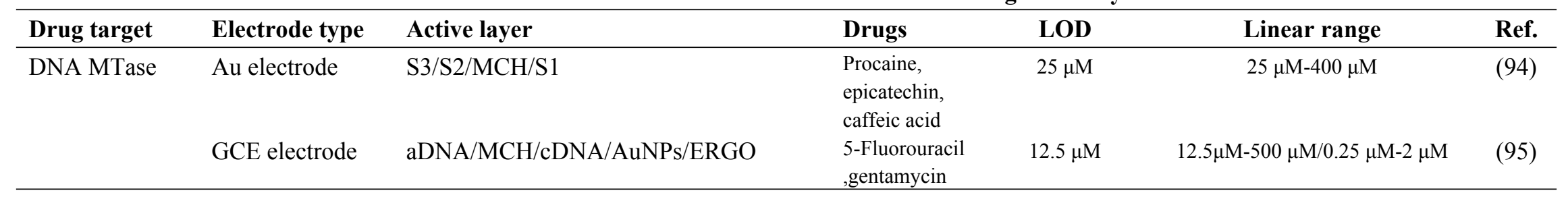




\begin{tabular}{|c|c|c|c|c|c|c|}
\hline & CP electrode & $\begin{array}{l}\text { BOD/HpaII/M.SssI/DNA2/MCH/DNA1/ } \\
\text { AuNP }\end{array}$ & $\begin{array}{l}\text { 5-Azacytidine, } \\
\text { epicatechin }\end{array}$ & $0.1 \mu \mathrm{M}$ & $0.1 \mu \mathrm{M}-2.5 \mu \mathrm{M} / 0.5 \mu \mathrm{M}-5 \mu \mathrm{M}$ & $(96)$ \\
\hline \multirow{3}{*}{ PKA } & GCE electrode & HRP/Phos-tag/P-Peptide/AuNPs/ & $\begin{array}{l}\text { Ellagic acid, } \\
\text { H-89 }\end{array}$ & $1.0 \mu \mathrm{M}$ & $1.0 \mu \mathrm{M}-10 \mu \mathrm{M} / 1.0 \mu \mathrm{M}-16 \mu \mathrm{M}$ & (97) \\
\hline & Au electrode & $\mathrm{GO}$ & H-89 & $0.01 \mu \mathrm{M}$ & $0.01 \mu \mathrm{M}-25 \mu \mathrm{M}$ & $(98)$ \\
\hline & Au electrode & P1-peptide & Ellagic acid & $1 \mu \mathrm{M}$ & $1 \mu \mathrm{M}-20 \mu \mathrm{M}$ & $(99)$ \\
\hline$\alpha$-Glucosidase & GE electrode & AgNPs/DA/PBA & $\begin{array}{l}\text { Gallic acid, } \\
\text { quercetin }\end{array}$ & $0.05 \mu \mathrm{M}$ & $0.5 \mu \mathrm{M}-100 \mu \mathrm{M} / 0.05 \mu \mathrm{M}-10 \mu \mathrm{M}$ & $(100)$ \\
\hline PARP & Au electrode & c-kit-1/MCH & $3-\mathrm{AB}$ & $1 \mathrm{nM}$ & $1 \mathrm{nM}-50 \mathrm{nM}$ & $(101)$ \\
\hline Thrombin & Au electrode & Carboxyl-group-free $\mathrm{Tb}$ peptides & Argatroban & $0.25 \mu \mathrm{M}$ & $0.25 \mu \mathrm{M}-8.0 \mu \mathrm{M}$ & $(102)$ \\
\hline HAT & Gold electrode & Coenzyme A aptamer & $\begin{array}{l}\text { Anacardic } \\
\text { acid, C646 }\end{array}$ & $0.01 \mu \mathrm{M}$ & $0.1 \mu \mathrm{M}-10^{3} \mu \mathrm{M} / 0.01 \mu \mathrm{M}-100 \mu \mathrm{M}$ & $(103)$ \\
\hline
\end{tabular}


Table S10. Photoelectrochemical biosensors for drug discovery

\begin{tabular}{|c|c|c|c|c|c|c|}
\hline Drug target & Electrode type & Active layer & Drugs & LOD & Linear range & Ref. \\
\hline \multirow[t]{2}{*}{ DNA MTase } & ITO electrode & ALP/AuNPs/DNA/Antibody/APTES & RG108 & $10 \mathrm{nM}$ & $10 \mathrm{nM}-1000 \mathrm{nM}$ & $(108)$ \\
\hline & ITO electrode & tDNA/BSA/pDNA-Au/CdSe/ $\mathrm{TiO}_{2}$ & Fisetin, 5-fluorouracil & $0.38 \mu \mathrm{M}$ & $0.38 \mu \mathrm{M}-400 \mu \mathrm{M}$ & $(109)$ \\
\hline PKA & ITO electrode & ALP/Phos-tag/P-Peptide/AuNPs-g- $\mathrm{C}_{3} \mathrm{~N}_{4}$ & HA-1077 & $0.1 \mu \mathrm{M}$ & $0.1 \mu \mathrm{M}-4 \mu \mathrm{M}$ & $(110)$ \\
\hline Thrombin & PI film electrode & MEA/MCH/peptide/gold NP & Argatroban & $0.5 \mathrm{nM}$ & $0.5 \mathrm{nM}-50000 \mathrm{nM}$ & $(111)$ \\
\hline T4 PNK & FTO electrode & $\begin{array}{l}\mathrm{CdSe}-\mathrm{DNA}_{2} / \mathrm{MCH} / \mathrm{DNA} 3 / \mathrm{CdS} / \\
\mathrm{g}-\mathrm{C}_{3} \mathrm{~N}_{4} / \mathrm{TiO}_{2}\end{array}$ & Adenosine diphosphate & $1.25 \mathrm{mM}$ & $1.25 \mathrm{mM}-5 \mathrm{mM}$ & $(112)$ \\
\hline
\end{tabular}


Table S11. Hybrid biosensors for drug discovery

\begin{tabular}{|c|c|c|c|c|c|}
\hline Biosensor type & Constitution & Detection biomarker & Drugs & Applications & Ref. \\
\hline FRET+CRET & $\begin{array}{l}\text { NIR semiconducting polymer PFODBT/ } \\
\text { cyanine dye (IR775S)/hydrophobic }\end{array}$ & ROS/RNS & isoniazid & hepatotoxicity & (124) \\
\hline
\end{tabular}


peroxyoxalate

Chemiluminesc

ent+fluorolumin

escent

Chemiluminesc

ent+fluorolumin

escent

FRET+AIE

FRET+AIE
Phenolate-dioxetane

derivative/casp3-cleavable peptide

Phenolate-dioxetane

derivative/hemi-cyanine

precursor/casp3-cleavable peptide

TPE-CHO/doxorubicin

H-PAMAM/doxorubicin
O2 - /caspase-3 activity

valproic acid

hepatotoxicity

(128)

$\mathrm{O} 2 \cdot-/ \mathrm{NAG} /$ caspase-3

cisplatin

nephrotoxicity loxorubicin

Efficacy

assessment

Efficacy
assessment

Efficacy
assessment

(137) 